\title{
Tornado-Like Vortices Above the Surface of a Liquid
}

\author{
Sinkevich OA* $^{*}$ \\ National Research University Higher School of Economics, Russia \\ *Corresponding author: Sinkevich OA, National Research University, Moscow Power Engineering Institute, 111250, Moscow, Russia
}

Submission: 眥 February 12, 2018; Published: 眥 August 31, 2018

\begin{abstract}
The shape of the funnel of a tornado-like vortex formed in a humid atmosphere above the water surface (lakes, seas, oceans) is discussed. An equation that describes the funnel shape of the tornado-like vortices is derived. We have modified the obtained funnel profiles and their dependence on the flow rate using the modified data of humidity changes with altitude in the free atmosphere. It was showed that the shape of the funnel depends on the turbulent air viscosity, the turbulent moisture diffusion coefficient, and the flow rate is obtained.
\end{abstract}

A vortex extended upward from the surface at least as far as cloud base (with that cloud base associated with deep moist convection), that is intense enough at the surface to do damage at one or more points along its path, should be considered a tornado.

Doswell C.D.III What is a tornado?

\section{Introduction}

\section{Characteristics of the turbulent flow of moist air in the funnel of tornado-like vortices}

At present, it is obvious that the problem of the tornadolike vortex is important not only for our planet to determine the conditions for the formation of a tornado-like vortex, it is required to take into account a number of hydrodynamic and plasma processes [1-6]. Along to prediction of a tornado-like vortex generation conditions [7-12] it is necessary to evaluate the characteristics of its quasi-stationary motion in a formed funnel: the mass of the moving moist air involved in the funnel and the size and form of the funnel. For a complete description of the phenomena, it is necessary to involve numerical calculations. We note that even for numerical calculations using powerful computers, the problem is very difficult because of the need to calculate multiphase turbulent flows with free, self-organizing boundaries $[1,6]$. However, "strict" numerical calculations, it is impossible to do without the use of many, often mutually exclusive, models. For example, how to choose an adequate model of turbulence (algebraic, k- $\varepsilon$ model, etc.) or the use of additional, often not accepted, hypotheses about certain processes used in calculations (mechanisms on the nature of moisture condensation, etc.). Therefore, along with numerical calculations of such flows, modeling problems that allow an exact solution and allow to determine the most important and observed characteristics of a tornado-like vortex.

In the literature, two commonly observed forms of the tornadolike vortex funnel are described: vague and dense. These forms, in turn, are divided into several types: snake-like, funnel-shaped, etc. Although in the course of its existence the tornado-like vortex can change the shape of the funnel, often, before it disappears, the tornado-like vortex funnel thins, becomes snake-like and then spreads out completely. In this paper, the stage of the formation of the funnel is not discussed, but the already existing form of the tornado-like vortex is considered (for example, tornado-like vortexes, in which the funnel widens toward the cloud, leaving into it, and narrows to the ground). Some data on the factors that affect the generation of tornado-like vortexes can be found in [1-5]. The problem of turning a vortex in a humid atmosphere in a tornadolike vortex capable of producing serious disruption on the path of its movement requires a separate analysis. An adequate analysis of the formation of a tornado-like vortex should show how, due to the development of instability, a funnel will form. In this paper, we do not consider the stage of tornado-like vortex formation, but investigate the mechanisms determining the shape of the formed tornado-like vortex funnel.

It was assumed in [7] that a vortex flow of a two-phase (dry air and a water vapors) media takes place in tornado-like vortex funnels. Funnel walls, according to the model [7], consist of hailstones, water droplets resulting from surface condensation of air moisture on wall surface. To describe the processes in the formed tornado-like vortex funnel, stationary Navier-Stokes equations, written in a cylindrical coordinate system for $V_{r}=0, V_{\varphi}(r), V_{z}(r, \varepsilon z), P(r, z), T(r, z)$, $\xi(\varepsilon r, z)<1, \varepsilon<<1$ using the small dimensionless parameter $\varepsilon=\frac{l r}{l z} \approx \frac{r_{r}}{h}<<1, \xi(\varepsilon r, z)<1$. Here $l r \approx r_{\mathrm{o}}, l z \approx h$ are characteristic dimensions of the system in radius and height directions, respectively. 
Using the presence of a small parameter, we can find solutions for all dependent variables in the next form $f(\varepsilon z, r)$. For this form of solution, the following hierarchy of derivatives holds for all dependent variables:

$$
\frac{\partial f}{\partial z} / \frac{\partial f}{\partial r} \approx \varepsilon
$$

The axisymmetric flow in the funnel is assumed to be subsonic, so the continuity equation takes the form

$$
\frac{1}{r} \frac{\partial}{\partial r} r V_{r}+\frac{\partial}{\partial z} V_{z}=\frac{1}{r} \frac{\partial}{\partial r} r V_{r}+\varepsilon \frac{\partial}{\partial z} V_{z}=0 .
$$

If $=V_{r}=0$, then, to within the parameter $\varepsilon<<1$, the continuity equation vanishes.

The projections of the equation of motion for unit directions have the form

$$
\begin{aligned}
& -\frac{V_{\phi}}{r}=-\frac{1}{\rho} \cdot \frac{\partial}{\partial} \frac{P}{r} \\
& \frac{1}{r} \frac{\partial}{\partial r} r \eta_{T} \frac{\partial}{\partial r} V_{\varphi}-\frac{V_{\varphi}}{r^{2}}=0 \\
& \frac{1}{r} \frac{\partial}{\partial r} r \eta_{T} \frac{\partial}{\partial r} V_{z}=\frac{\partial}{\partial} \frac{P}{z}+\rho g
\end{aligned}
$$

The energy equation has the form

$$
\frac{1}{r} \frac{\partial}{\partial r} r \lambda_{T} \frac{\partial}{\partial} \frac{T}{r}+q_{v}=0
$$

Equation of state of air with water vapor:

$$
P=P_{a}+P_{w}=R_{\mu} \rho T(1+\xi(\varepsilon r, z)) \quad P=P_{a}+P_{w}=R_{\mu} \rho T(1+\xi(\varepsilon r, z))
$$

$\operatorname{Here} \mathrm{V}\left(0, \mathrm{~V}_{\varphi}, \mathrm{V}_{\mathrm{z}}\right)$ is the velocity field, $\mathrm{r}, \mathrm{z}$ are the radial and vertical coordinates, respectively, $\mathrm{V}_{\varphi}$, is the angular velocity component, $V_{\mathrm{z}}$ is the axial velocity component, $\eta_{\mathrm{T}}=$ const is the coefficient of turbulent dynamic viscosity, $\mathrm{P}$ is the air pressure, $\rho$ is the air density, $\mathrm{T}$ is the temperature, $\mathrm{g}$ is an acceleration of gravity, $\mathrm{R} \mu=176.846 \mathrm{~J} /$ $\mathrm{kg} \mathrm{K}$ is the gas constant of the mixture consisting of air and water vapor, $\lambda_{\mathrm{T}}=$ const is the turbulent thermal conductivity of air in the tornado-like vortex funnel, qv is the volumetric heat release, $\xi$ is the humidity of air, so above equations were supplemented by the equation of the humidity diffusion in the funnel

$$
\frac{1}{r} \frac{\partial}{\partial r} r D r \frac{\partial}{\partial} \frac{\xi}{r}+\frac{\partial}{\partial} D z \frac{\partial}{\partial} \frac{\xi}{z}=V z \frac{\partial}{\partial} \frac{\xi}{z}
$$

Dr, Dz are the turbulent radial and axial coefficients of diffusion of moisture in the funnel.

The following boundary conditions were used for the components of the velocity vector, the temperature of the medium and the moisture content:

$$
\begin{gathered}
V_{\varphi}(r=0)=0, V_{\varphi}\left(r=r_{\grave{\mathrm{O}}}\right)=r_{\mathrm{o}} \Omega, \Omega=\mathrm{const} \frac{\partial}{\partial r} V_{z}(r=0)=0, V_{z}\left(r=r_{\mathrm{o}}\right)=0 \\
\left(\frac{\partial}{\partial r} T\right)_{r=0}=0 T\left(r=r_{\grave{0}}\right)=T W
\end{gathered}
$$

$\left(\frac{\partial}{\partial} \frac{\xi}{r}\right)_{r=0}=0, \xi\left(r=r_{\grave{o}}\right)=\xi_{w}, \xi(r, z=0)=\xi_{0}=$ const
Considering the change of pressure with an altitude $\mathrm{z}$ $P(r, \varepsilon z)=P_{0}(r, z)+R_{\mu} \rho T \xi(\varepsilon z)$. The dependence of 3 on the coordinate is found from equation

$$
\rho g=-\frac{\partial P_{0}}{\partial z}, \frac{\partial}{\partial} \frac{P_{0}}{r}=\Omega r
$$

Using this representation for the pressure from (3) we obtain the following equation for the vertical velocity of the moist air movement

$$
\frac{v_{T}}{r} \frac{\partial}{\partial r} r \frac{\partial}{\partial r} V_{z}=R_{\mu} T \frac{\partial}{\partial} \frac{\xi}{z}
$$

Assuming that in the presence of gravity, the pressure gradient in the axial direction is determined by the air humidity gradient (9), and using the boundary conditions (7), we represent the solution of equation (11) in the following form

$$
V_{z}(r)=V_{z}(0)\left(1-\frac{r^{2}}{r_{\grave{o}}^{2}}\right)
$$

Here $v_{\grave{o}}=\eta_{\grave{\mathrm{o}}} / \rho=$ const, is the coefficient of turbulent viscosity. The velocity on the axis of the tornado-like vortex funnel is

$$
V_{z}(0)=\frac{R \mu T(0) r_{\mathrm{O}}^{2}}{4 v_{\mathrm{o}}}\left|\frac{\partial \xi}{\partial z}\right|
$$

It is easy to see from equation (13), the velocity $V_{z}(0)$ is depending on the gradient of vertical humidity $\left|\frac{\partial \xi}{\partial z}\right|$, which could be found from the solution of the diffusion equation for water vapor (6). Some, more detailed calculations of processes in the tornado-like vortex funnel, performed within the framework of the model proposed here, can be found in our works [7-11]. Here we will look for the shape of a steady tornado-like funnel, using the presence of the small parameters introduced above. It is believed that air humidity changes insignificantly along the radius of the funnel (see equation (5)), therefore, when solving the diffusion equation, moisture transfer in the radial direction can be neglected $\frac{1}{r} \frac{\partial}{\partial r} r D r \frac{\partial}{\partial} \frac{\xi}{r} / V z \frac{\partial}{\partial} \frac{\xi}{z} \approx \varepsilon^{2}<<1$. In the same approximation with respect to the parameter $\varepsilon<<1$, we replace in equation (6) $V_{z}(r)$ by $V_{z}(0)$.The solution of equation (6) with epy boundary condition (7) can be represented in the form:

$$
\xi(z)=\xi_{0} \exp \left(\frac{-z D \text { ò }}{r_{\mathrm{O}}^{2} V_{z}(0)}\right) \text {. }
$$

Having found from equation (14) the gradient of vertical humidity $|\partial z|$, it is possible to present a vertical flow velocity profile in a tornado-like vortex funnel in the form:

$$
V_{z}(r)=\left(\frac{\xi_{0} D_{\mathrm{o}} T(0) R_{\mu}}{4 v_{\grave{\mathrm{o}}}}\right)^{1 / 2}\left(1-\frac{r^{2}}{r_{\grave{\mathrm{o}}}^{2}}\right) \exp \left(-\frac{\pi \rho_{0} D_{\mathrm{o}} z}{4 G}\right)
$$

Here $\mathrm{G}$ is a flow rate of moist air in the funnel, $\xi 0$ is the humidity at the base of the tornado-like vortex.

The mass air flow in the tornado-like vortex is found from the equation: 


$$
G=2 \pi \int_{0}^{r_{\grave{o}}} \rho_{0} V_{z} r d r
$$

Substituting solution (15) into this equation and integrating, we find the relationship between the flow rate, the funnel radius and the humidity change in height:

$$
G=\frac{\pi \rho_{0} r_{\dot{\mathrm{o}}}^{2}}{2}\left(\frac{\xi_{0} D_{\mathrm{o}} \mathrm{R}_{\mu} T(0)}{4 v_{\dot{\mathrm{o}}}}\right)^{1 / 2} \exp \left(\frac{-z \pi \rho_{0} D_{\dot{\mathrm{o}}}}{4 G}\right)=\text { const }
$$

In the considered tornado-like vortex funnel model it was assumed that the funnel walls are impermeable to air, so the flow rate should remain constant in height. As can be seen, formula (16) relates the air flow rate in a tornado-like vortex and the funnel radius.

$$
r_{\dot{\mathrm{o}}}=\left(2 G / \pi \rho_{0}\right)^{1 / 2}\left(\frac{\xi_{0} D_{\mathrm{o}} \mathrm{R}_{\mu} T(0)}{4 v_{\grave{\mathrm{o}}}}\right)^{-1 / 4} \exp \left(\frac{z \pi \rho_{0} D_{\mathrm{o}}}{8 G}\right)
$$

So if the flow rate remains constant the funnel radius should vary with altitude Next, we use this connection to represent the variation in the tornado-like vortex funnel radius with height. From the equations obtained it can be seen that, with a constant air flow, the change in the radius of the tornado funnel is associated with a change in the humidity of the air along the height. However, it is difficult to directly use the formulas obtained because the turbulent coefficients are poorly known and vary in different ways in a calm atmosphere and in the development of a tornado. Recently in the change of humidity with altitude (the city of Kirensk, Russia) were obtained. For getting the most appropriate tornado-like vortex funnel shape, we approximated a change of humidity with altitude by the next function corresponding to the data from [12]:

$$
\xi(z)=\xi_{0} \exp \left(-b_{a} z\right)
$$

Here $b_{a}=2.839 \cdot 10^{-4} 1 / \mathrm{m}$ is the value obtained by approximating the data.

Further, solutions $(12,13)$ and the formula for the humidity change with altitude (18) will be used for finding a change of the funnel radius with altitude. It should be noted that the data given in and approximated by formula (18) refer to the free atmosphere. Using the formula (18) suitable to the free atmosphere for a tornado-like vortex funnel, it can be assumed that a more intense change of moisture along the height occurs in the tornado-like vortex funnel $\left(b_{\dot{\mathrm{o}}}>2.839 \cdot 10^{-4} 1 / \mathrm{m}\right)$. Therefore, to compare the shapes of the tornado-like vortex showers shown in Figure 2 with the theoretical data, this circumstance should be taken into account. Using distributions of pressure, density, and moisture in the form (14) we represent the change of the funnel radius in height in the form

$$
r_{\mathrm{o}}=\frac{\Omega \exp \left[\left(b_{\mathrm{o}} z\right) / 2\right]}{a^{2} M}\left(\frac{8 v_{\mathrm{o}} \gamma G}{\pi \rho(0) b_{\dot{\mathrm{o}}} \xi_{0}}\right)^{1 / 2}
$$

Formula (19) allows us to calculate the average radius of the funnel of a tornado-like vortex

$$
\left\langle r_{\mathrm{o}}\right\rangle=\frac{1}{h} \int_{0}^{h} r_{\mathrm{o}}(z) d z=\frac{2 \Omega}{b_{\grave{\mathrm{o}}} h a^{2} M}\left[\exp \left(\frac{b_{\mathrm{o}} h}{2}\right)-1\right]\left(\frac{8 v_{\mathrm{o}} \gamma G}{\pi \rho(0) b_{\mathrm{o}} \xi_{0}}\right)^{1 / 2}
$$

and establish a scaling between this radius and other parameters of the steady tornado-like vortex.

Here $\mathrm{a}$ is the speed of sound $\boldsymbol{r}_{T}, \mathrm{~h}$ are the funnel radius and its height, respectively $\mathrm{M}$ is the Mach number for the flow in the funnel, $\gamma$ is the adiabatic index of moist air, $\Omega=$ const is an angular velocity of the medium rotation

If the inequality $\frac{b_{\grave{0}} h}{2}>>1$ is satisfied, then the complex $\frac{2 \Omega}{a^{2} M}\left(\frac{8 v_{\dot{\partial}} \gamma G}{\pi \rho(0) \xi_{0} b T}\right)^{1 / 2}$, entering into the equation (20) can be represented in the form:

$$
\frac{2 \Omega}{a^{2} M}\left(\frac{8 v_{\mathrm{o}} \gamma G}{\pi \rho(0) b_{\mathrm{o}} \xi_{0}}\right)^{1 / 2}=b_{\mathrm{o}} h\left\langle r_{\mathrm{o}}\right\rangle \exp \left(\frac{-b_{\mathrm{o}} h}{2}\right)
$$

This allows us to present a change in the radius of the funnel of a steady tornado-like vortex with a height in the form:

$$
r_{\mathrm{O}}(z)=b_{\grave{\mathrm{o}}} h\left\langle r_{\mathrm{O}}\right\rangle \exp \left(\frac{b_{\grave{\mathrm{o}}}(z-h)}{2}\right)
$$

Using the observational data on the shape of the tornadolike vortex funnel with height, we can calculate, using formula (21), a parameter characterizing the change in humidity inside the tornado-like vortex funnel. A comparison of the results of calculating the shape of the tornado funnel with height according to formula (21) with the data of observations of real tornadoes $[13,14]$ is presented in our work [15].

\section{Conclusion}

An equation that describes the shape of the tornado-like vortex funnel, depending on the turbulent air viscosity, the turbulent moisture diffusion coefficient, and the flow rate is obtained.

Now we could obtain funnel profiles of the tornado-like vortices and find their dependence on the flow rate using the modified data of humidity changes with altitude in the free atmosphere. This approach allows eliminating some unknown turbulent characteristics of flow inside the funnel. These results give the funnel shape and its dependence on the tornado-like vortex intensity that is closest to the observation data.

\section{References}

1. Church C, Burgess D, Doswell C (2013) The tornado: its structure, dynamics, prediction and hazards. Geophysical Monograph Series, $\left(1^{\text {st }}\right.$ edn), Vol 69, American Geophysical Union, USA.

2. Levens PJ, Labrosse N, Fletcher L, Schmieder BA (2015) Solar tornado observed by EIS plasma diagnostics. Astronomy and Astrophysics 582: A27.

3. Doswell CA (2005) Progress toward developing a practical societal response to severe convection. EGU Sergei Soloviev Medal Lecture. Nat Hazards Earth Syst Sci 5: 1-12. 
4. Varaksin AY, Romash ME, Kopeytsev VN (2011) Tornado. FIZMATLIT, Moscow, Russia, p. 4.

5. Kushin VV (1987) Gravitational-thermal processes in tornadoes. Tziiatominform.

6. Sinkevich OA, Maslov SA, Zade NG (2017) Role of electric discharges in the generation of atmospheric vortices. Plasma Physics Reports 43(2): 232-252.

7. Sinkevich OA (1996) Flow of the tornado funnel including phase transitions high temperature. IOP Science 34(6): 922-927.

8. Sinkevich OA, Chikunov SE (1994) Pros. of the $1^{\text {st }}$ All-Russian Conf. Heat and Mass Transfer, Moscow, Russia 4: 360-363.

9. Sinkevich OA, Glazkov VV, Smirnov EP (1996) Multiphase energy and mass transfer in atmosphere and mechanism of tornado formation. Heat Transfer Research 27(1): 190-194.
10. Sinkevich OA (2005) Pros. of the $2^{\text {nd }}$ All-Russian Conf. Heat and Mass transfer in Swirling Flows, Moscow, Russia, pp. 51-52.

11. Sinkevich OA, Chikunov SE, Glazkov VV (2010) Processes in the twophase layer near the heathed surface of the ocean and generation of atmospheric vortices. Heat Transfer Research 41(1): 75-92.

12. Komarov VS, Ilyin SN, Lomakina NY, Nakhtigalova DP (2013) Features of the vertical structure of the temperature and humidity fields in the boundary layer of the atmosphere over eastern siberia, depending on its cloud state. Part 1. Phase characteristics and variability. Optics of the Atmosphere and the Ocean 3: 26-30.

13. http://tornadotitans.com

14. https://fineartamerica.com/profiles/ethan-schisler.html

15. Sinkevich OA, Bortsova AA (2017) Two-phase flows in the formed tornado funnel. IOP Conf Series: Journal of Physics: Conf Series 891: 012027.
Creative Commons Attribution 4.0 International License

For possible submissions Click Here

\section{Submit Article}

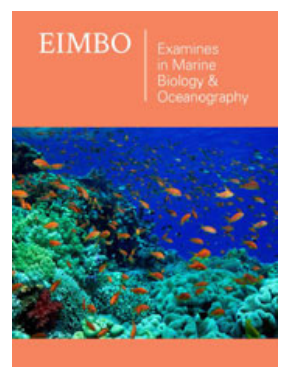

Examines in Marine Biology \& Oceanography

\section{Benefits of Publishing with us}

- High-level peer review and editorial services

- Freely accessible online immediately upon publication

- Authors retain the copyright to their work

- Licensing it under a Creative Commons license

- Visibility through different online platforms 